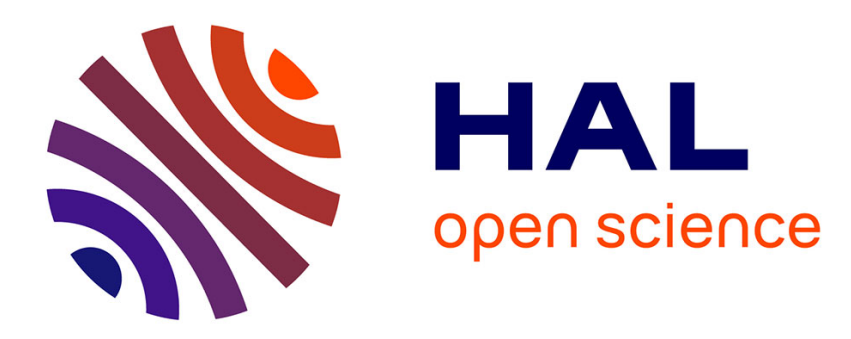

\title{
Modélisation de moteurs piézoélectriques
}

\author{
M. Brissaud
}

\section{To cite this version:}

M. Brissaud. Modélisation de moteurs piézoélectriques. Journal de Physique IV Proceedings, 1994, 04 (C5), pp.C7-237-C7-240. 10.1051/jp4:1994547 . jpa-00253042

\section{HAL Id: jpa-00253042 https://hal.science/jpa-00253042}

Submitted on 1 Jan 1994

HAL is a multi-disciplinary open access archive for the deposit and dissemination of scientific research documents, whether they are published or not. The documents may come from teaching and research institutions in France or abroad, or from public or private research centers.
L'archive ouverte pluridisciplinaire HAL, est destinée au dépôt et à la diffusion de documents scientifiques de niveau recherche, publiés ou non, émanant des établissements d'enseignement et de recherche français ou étrangers, des laboratoires publics ou privés. 


\title{
Modélisation de moteurs piézoélectriques
}

\author{
M. BRISSAUD
}

INSA, Laboratoire de Génie Electrique et Ferroélectricité, 69621 Villeurbanne cedex, France

\begin{abstract}
This paper deals with to the theoretical analysis of two kinds of piezoelectric motors in order to determine for each of them the dedicated electromechanical coupling factors (EMC factors) which are important choice criteria for the energy conversion inside the piezoceramic materials. The fist motor is based on the non-symmetrical radial motion of a piezoelectric disk. The second type of motor uses Lamb wave propagation inside a piezoceramic disk. The EMC factor determination implies the calculation of the displacements, stresses and electrical impedance of each type of motors. As a result, the rotor in contact with the motor stator rotates because an elliptic motion occurs on the surface situated between the rotor and the stator. The analytical modellings show that the energy conversion is characterized by one EMC factor for the first type of motor and two EMC factors for the second type. However, in each case, two kind of waves are involved : longitudinal and shear waves. These results are consistent with the constitutive piezoelectric equations used for each of the considered modes.
\end{abstract}

\section{I - INTRODUCTION}

De nombreux types de moteurs piézoélectriques ont été imaginés et entrent maintenant dans de nombreuses applications [1]-[4]. Ils sont basés soit sur l'interaction d'une onde longitudinale et d'une onde transversale qui pour certains modes particuliers sont couplées [5] soit sur l'utilisation d'ondes de Lamb [6] pour lesquelles des ondes de mode longitudinales et transversales sont animées de la même vitesse [7]. Le premier type qui met en jeu des modes de contour à symétrie uniaxiale repose sur les travaux de LOVE [5] puis de HOLLAND [7] et d'ONOE [8]. Les seconds sont basés sur les travaux de LAMB et de VIKTOROV [6].

De nombreux auteurs ont récemment étudié ces différents types de moteurs en supposant soit que les disques piézoélectriques sont isotropes et la piézoélectricité n'intervient pas soit en considérant seulement l'effet inverse de la piézoélectricité. De ce fait il n'est pas possible d'obtenir les amplitudes des contraintes et des déformations en fonction des coefficients piézoélectriques [7]. Par suite ces modèles sont incapables de définir pour une structure donnée, quel est le coefficient de couplage électromécanique correspondant.

Le but de ce travail est la détermination des différents coefficients de couplage électromécaniques intervenant dans deux types importants de moteurs. Dans chaque cas il est nécessaire de calculer les champs de contraintes et de déplacements dans les structures considérées ce qui permet ensuite la détermination de l'expression de leur impédance électrique. Après la description des structures et des hypothèses utilisées nous donnerons pour chacun des moteurs les valeurs théoriques des divers coefficients de couplage ainsi qu'une comparaison entre les résultats expérimentaux et théoriques issus de la simulation.

\section{II - STRUCTURES DES MOTEURS}

La figure 1 donne la structure du premier type de moteur. Le stator est constitué par un disque piézoélectrique épais polarisé axiallement. Une des électrodes recouvre entièrement une des faces alors que l'autre électrode est découpée en secteurs (4 sur la figure). Chaque paire de secteurs est alimentée par respectivement des tensions sinusoïdales de la forme $\pm \sin \omega t$, et $\pm \cos \omega t$. Il s'ensuit que les déformations latérales créées par chaque couple opposés de secteurs sont à la même fréquence mais sont déphasées dans l'espace et dans le temps de $\pi / 2$. Elles engendrent une déformation résultante elliptique qui pourra entraîner un rotor si celui-ci est en contact avec le stator [1]-[4].

L'anneau mince de la figure 2 correspond au stator du second type de moteur. L'électrode supérieure de l'anneau mince polarisé axialement est divisée en deux groupes de secteurs séparés par une distance égale à $\lambda / 4$ (ou $3 \lambda / 4$ à la partie supérieure). Les deux groupes de secteurs sont alimentés par des tensions déphasées d'un quart de période. Chaque groupe engendre une onde de Lamb stationnaire et la résultante de ces deux ondes stationnaires déphasées et décalées dans l'espace 
est une onde de Lamb progressive qui entraîne un rotor s'il est placé sur l'une ou l'autre des faces de l'anneau piézoélectrique du stator [6]-[9].

Pour les deux types de moteur nous restreindrons notre étude au cas du stator seul et nous déterminerons dans chaque cas l'impédance électrique d'un secteur (figures 1 et 2 ).

\section{III - MODÉlISATION DU PREMIER TYPE DE MOTEUR}

Les principaux résultats théoriques concernant ces moteurs sont issus des travaux relatifs aux vibrations libres de contours de disques isotropes minces [5], [7], [8]. Pendant longtemps ces modes ne furent pas utilisés car il n'existait pas de méthode pour les exciter physiquement. L'apparition des matériaux piézoélectriques en permettant au moyen d'électrodes judicieusement placées, d'exciter un grand nombre de ces modes ont rendu possible leur utilisation dans divers types de moteur piézoélectrique. TAKANO et al ont été parmi les premiers à introduire la piézoélectricité dans l'analyse de ces modes pour réaliser des moteurs [4]. Cependant leur analyse est incomplète et limitée car ils négligent l'effet piézoélectrique direct et considère que le matériau est isotrope. Il n'est alors pas possible d'exprimer les amplitudes des déplacements et des contraintes en fonction du courant d'alimentation et des paramètres du matériau et d'en déduire le coefficient de couplage électromécanique de ces modes.

\section{1 - Moteur du premier type (figure 1)}

Considérons le disque piézoélectrique de rayon a de la figure 1. Si son épaisseur est petite par rapport à son rayon les fréquences de résonance du mode radial seront situées bien au-dessous du mode en épaisseur. On pourra, en première approximation supposer que ces modes sont découplés c'est-à-dire T33 \# 0. De plus si on néglige les effets de bords entre secteurs, les équations d'états piézoélectriques se réduisent à :

$\mid \begin{aligned} & T_{\mathrm{rr}}=C_{11}^{\mathrm{D}} \mathrm{S}_{\mathrm{r}}+\mathrm{C}_{12}^{\mathrm{D}} \mathrm{S}_{\theta}-\mathrm{h}_{31} \mathrm{D}_{3} \\ & \mathrm{~T}_{\theta \theta}=\mathrm{C}_{12}^{\mathrm{D}} \mathrm{S}_{\mathrm{r}}+\mathrm{C}_{11}^{\mathrm{D}} \mathrm{S}_{\theta}-\mathrm{h}_{31} \mathrm{D}_{3}\end{aligned}$

$E_{3}=-h_{31}\left(S_{\tau}+S_{\theta}\right)+\beta_{33}^{S} D_{3}$ et $\quad \mid \begin{aligned} & \mathrm{T}_{\mathrm{r} \theta}=\mathrm{C}_{66}^{\mathrm{D}} \mathrm{S}_{\mathrm{r} \theta} \\ & \mathrm{T}_{\mathrm{zz}}=\mathrm{T}_{\mathrm{z} \theta}=\mathrm{T}_{\mathrm{rz}}=0\end{aligned}$

et $\quad E_{1}=E_{2}=0$ et $D_{1}=D_{2}=0$

L'induction $D_{3}$ est prise comme variable indépendante et s'écrit : $D_{3}=D(r) \cos n \theta$

L'ensemble des équations (1) et (2) a une solution non nulle si les déplacements $u_{r}$ et $u_{\theta}$ sont la somme de déplacements de modes longitudinaux (radial) et transversaux situés dans le plan $r \theta$ du disque soit [10]-[11] :

$$
\mid \begin{aligned}
& u_{r}=\left[A_{n} J_{n}^{\prime}(h r)+B_{n} J_{n}(k r) / r\right] \cos n \theta=U_{r} \cos n \theta \\
& u_{\theta}=-\left[A_{n} J_{n}(h r) / r+B_{n} J_{n}^{\prime}(k r)\right] \sin n \theta=U_{r}^{\prime} \sin n \theta
\end{aligned}
$$

où $n$ est un nombre entier et $\theta$ est l'angle entre secteurs en coordonnées polaires.

$\mathrm{J}$ et $\mathrm{J}_{\mathrm{n}}^{\prime}$ sont respectivement les fonctions de Bessel de première espèce d'ordre $\mathrm{n}$ et leurs dérivées, $\mathrm{h}=\omega / \mathrm{V}_{\mathrm{L}}, \mathrm{k}=\omega / \mathrm{V}_{\mathrm{T}}$ avec $V_{L}^{2}=C_{11}^{D} / \rho, V_{T}^{2}=C_{66}^{D} / \rho, V_{L}$ et $V_{T}$ sont respectivement les vitesses des ondes de modes longitudinal et transversal dans le plan $\mathbf{r}-\theta$.

L'introduction des conditions aux limites dans (1) conduit à un système linéaire en $S_{r}$ et $S_{\theta}$ qui permet de calculer les amplitudes $A_{n}$ et $B_{n}$ des deux types d'ondes [11].

L'impédance électrique se déduit de l'équation (2) en intégrant les charges sur la surface du secteur et le champ électrique selon son épaisseur. On obtient une expression de la forme $[10]: Z=\left[1-k_{S}^{2} K / \Delta\right] / j C_{o} \omega$

où $\mathrm{C}_{\mathrm{O}}$ est la capacité du secteur libre, $\mathrm{K}$ est une expression liée aux fonctions de Bessel et $\mathrm{k}_{\mathrm{S}}$ est le coefficient de couplage correspondant à ce moteur. Il s'écrit : $\mathrm{k}_{\mathrm{S}}^{2}=\mathrm{k}_{31}^{2} / \varepsilon_{33}^{\mathrm{S}} \mathrm{C}_{66}^{\mathrm{D}}=2 \mathrm{k}_{31}^{2} / 1-\sigma^{\mathrm{D}}$

où $\sigma^{D}=C_{12}^{D} / C_{11}^{D}$. Ce rapport est homogène à un coefficient de Poisson [11]. L'expression (7) est similaire à celle donnant le coefficient de couplage du mode radial d'un disque mais les paramètres qui y interviennent sont différents [12].

Il faut noter ici que, bien que deux ondes interviennent dans le fonctionnement (longitudinal (h) et transversal (k)), il n'y a qu'un seul coefficient de couplage. Cela provient du fait que dans l'expression de la contrainte de cisaillement $\mathrm{T}_{\mathrm{r} \theta}$ il n'y a pas de terme piézoélectrique alors qu'il y en a un dans $T_{\mathrm{rr}}$ et $\mathrm{T}_{\theta \theta}$. Cela conduit à un découplage des deux modes.

\section{2 - Moteur du deuxième type (figure 2)}

Pour ce type de moteur les déplacements s'effectuent selon $\theta$ et selon $\mathrm{z}$.

On considère généralement que $u_{\theta}$ et $u_{Z}$ dérivent de potentiels scalaires et vecteurs harmoniques [6] et on peut mettre $\mathrm{u}_{\theta}$ et $\mathrm{u}_{\mathrm{Z}}$ sous la forme : 


$$
\mid \begin{aligned}
& u_{\theta}=U(z) \exp j(\omega t-k r \theta)=A \operatorname{ch~qz} e^{j x}+B \operatorname{shqz} e^{j x} \\
& u_{z}=W(z) \exp j(\omega t-k r \theta)=D \operatorname{sh~sz~} e^{j x}+C \operatorname{chsz} e^{j x}
\end{aligned} \text { et } x=k r \theta
$$

avec

$$
\begin{array}{|l|l|l}
\mathrm{q}^{2}=\mathrm{k}^{2}-\mathrm{k}_{\mathrm{L}}^{2} & (9) & \begin{array}{l}
\mathrm{k}_{\mathrm{L}}=\omega / \mathrm{V}_{\mathrm{L}} \\
\mathrm{k}_{\mathrm{T}}=\omega / \mathrm{V}_{\mathrm{T}}
\end{array}
\end{array} \quad \begin{aligned}
& \mathrm{V}_{\mathrm{L}}^{2}=\mathrm{C}_{33}^{\mathrm{E}} / \rho \\
& \mathrm{s}^{2}=\mathrm{k}^{2}-\mathrm{k}_{\mathrm{T}}^{2}=\mathrm{C}_{55}^{\mathrm{E}} / \rho
\end{aligned}
$$

Nous supposerons qu'en première approximation $\mathrm{u}_{\theta}$ et $\mathrm{u}_{\mathrm{Z}}$ ne dépendent pas de $\mathrm{r}$ donc $\mathrm{r}=\mathrm{cte}$ dans (8). Les contraintes $\mathrm{T}_{\theta \theta}$, $\mathrm{T}_{\mathrm{zZ}}$ et $\mathrm{T}_{\mathrm{rz}}$ se réduisent alors à

$$
\mid \begin{aligned}
& T_{\theta \theta}=C_{11}^{E} S_{\theta \theta}+C_{33}^{E} S_{z z}-e_{31} E_{3} \\
& T_{z z}=C_{13}^{E} S_{\theta \theta}+C_{33}^{E} S_{z z}-e_{31} E_{3} \\
& T_{\theta z}=C_{33}^{E} S_{\theta z}-e_{15} E_{1}
\end{aligned}
$$

Compte tenu des relations (9) à (11) et des conditions aux limites (contraintes nulles sur les faces supérieures et inférieures de l'anneau) les expressions des contraintes $T_{\theta \theta}, \mathrm{T}_{Z z}$ et $\mathrm{T}_{\theta z}$ conduisent à un système linéaire de dimension $4 \times 4$ que l'on peut scinder en deux sous systèmes. La résolution de ce système permet d'exprimer $\mathrm{A}, \mathrm{B}, \mathrm{C}, \mathrm{D}$ en fonction des déterminants de chaque sous système et de $E_{3}$ et $E_{1}$ les 2 composantes du champ électrique entre les deux électrodes. En calculant ensuite la charge totale sur la surface de l'électrode et en intégrant le champ électrique suivant l'épaisseur du matériau on obtient l'expression de l'admittance électrique. Il a la forme suivante:

$$
\mathrm{Y}=\mathrm{j} \mathrm{C} \mathrm{C}\left[1+\mathrm{k}_{\mathrm{c}}^{2} \mathrm{~K}_{\mathrm{c}}+\mathrm{k}_{\mathrm{L}}^{2} \mathrm{~K}_{\mathrm{L}}\right]
$$

où $C_{o}=\varepsilon_{33}^{S} a R \theta_{0} \sin \left(\alpha R \theta_{0} / 2\right) / 2 d$ est la capacité du secteur

$\mathrm{K}_{\mathrm{L}}$ et $\mathrm{K}_{\mathrm{C}}$ sont des termes liés respectivement aux amplitudes des ondes longitudinales et transversales dans le matériau.

$$
k_{c}^{2}=\left|e_{31} e_{33}\right| / \varepsilon_{33}^{S} C_{55}^{E} \text { et } k_{L}^{2}=e_{33}^{2} / \varepsilon_{33}^{S} C_{33}^{E}
$$

Les coefficients $k_{c}^{2}$ et $k_{L}^{2}$ sont les coefficients de couplage relatifs aux deux types d'ondes. Ces coefficients caractérisent la conversion de l'énergie au sein du matériau. Dans ce type de moteur les deux types d'ondes intervenant de façon similaire dans les expressions (9) des contraintes il est normal d'avoir deux coefficients de couplage. De plus ils sont différents de ceux établis précédemment. Une approche par éléments finis a montré que le fonctionnement de ce moteur est intimement lié aux modes latéraux de type "31" ce que confirme bien le coefficient de couplage $\mathrm{k}_{\mathrm{c}}$ [8]. Cependant l'expression de $\mathrm{k}_{\mathrm{c}}$ montre que ce mode résulte de linteraction des modes latéraux (e31) et de cisaillement $\left(\mathrm{C}_{55}^{\mathrm{E}}\right)$.

\section{IV - RÉSULTATS EXPÉRIMENTAUX}

La figure 3 montre, pour le premier type de moteur, l'évolution de l'impédance électrique d'un secteur en fonction de la fréquence. Le disque piézoélectrique de type PZT a un diamètre de $25 \mathrm{~mm}$ et une épaisseur de $5 \mathrm{~mm}$. L'électrode supérieure est divisée en quatre secteurs. La figure 4 montre l'évolution théorique de l'impédance du secteur précédent lorsque les deux premiers modes sont pris en compte. La résonance principale ( $2^{\mathrm{e}}$ pic) correspond au premier mode alors que les premiers et troisièmes pics correspondent au second mode. Les différences entre les deux courbes proviennent de l'épaisseur du disque qui n'est pas petite par rapport à son épaisseur et des effets de bords entre secteurs qui introduisent des termes supplémentaires dans les expressions (1) et (2) et modifient un peu les conditions de résonance de l'ensemble.

\section{$\mathrm{V}$ - CONCLUSION}

Nous avons montré que l'on pouvait obtenir les expressions des coefficients de couplage spécifiques à chacun des types de moteurs piézoélectriques modélisés. Ces coefficients sont différents de ceux établis pour les modes de vibration simples des éléments piézoélectriques. Ils sont un critère important pour le choix du matériau piézoélectrique réalisant la conversion de l'énergie dans les moteurs.

\section{BIBLIOGRAPHIE}

[1] UEHA S. et al - Ultrasonics Motors. Ultrasonics International 85. London 1985.

[2] SHINSEE KOGYO K. - Motor for utilizing ultrasonic wave oscillation. Pat. $n^{\circ} 61,277384,1985$.

[3] UEHA S., UKITA A., KUROSAWA M., MORI E. - Ultrasonic actuator using the extensional vibration of cylindrical shell. Ultrasonics International 87. London, 1987.

[4] TAKANO T. et al - Analysis of non axisymmetric vibration mode piezoelectric annular, plate and its application to an ultrasonic motor. IEEE Trans. U.F.F.C. Vol. 37, n 6, Nov. 1990. 
[5] LOVE A.E.H. - Mathematical theory of elasticity. Dover, New-York 1944.

[6] VIKTOROV I. A. - Rayleigh and Lamb waves Plenum Press 1967.

[7] HOLLAND R. - Numerical studies of elastic-disk contour modes lacking axial symmetry. J. Acoust. Soc. Am. Vol. 40, n 5 , April 1966.

[8] ONOE M., KURACHI T. - Non-axisymmetric vibrations of a circular ceramic disk. IEEE Trans. S. U. Vol. 49, $n^{\circ} 1$, 1966.

[9] CLAYSSEN F., Le LETTY R., HAMONIC B., BOSSUT R. - Analyse d'un moteur ultrasonore piézoélectrique à l'aide de la modélisation. Sup. J. Physique III. Vol. 2, 1992.

[10] BRISSAUD M., DION J. L., EYRAUD L. - Double action piezoelectric motors. 4 th Europ. Freq. and Time Forum. Neuchâtel. March 1990.

[11] BRISSAUD M. - Analytical modelling of an ultrasonic motor. UI'93. Wien. Juillet 1993.

[12] IEEE. Standards on Piezoelectricity. Special issue of Trans. SU. Vol. 31, n² 2, Pt II. May 1984.

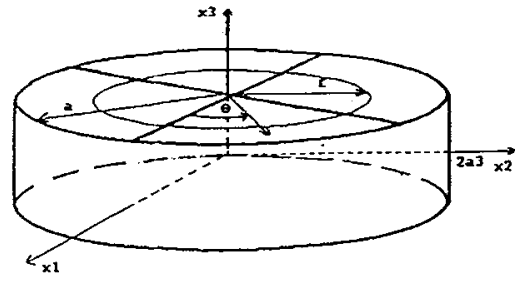

Fig. 1 Moteur à couplage de -modes (1 $1^{\mathrm{er}}$ type)

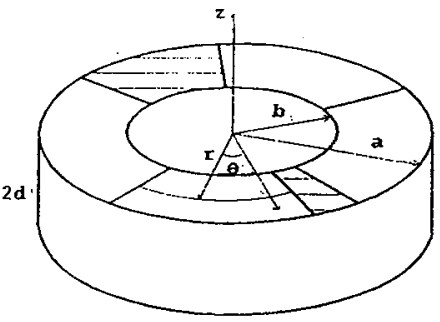

Fig. 2 Moteur à onde de Lamb (2c type)

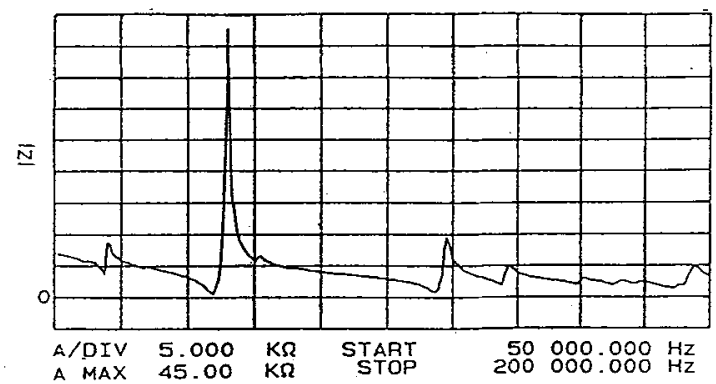

Fig. 3 Évolution expérimentale, en fonction de la fréquence, de l'impédance d'un secteur du stator du moteur du 1 er type

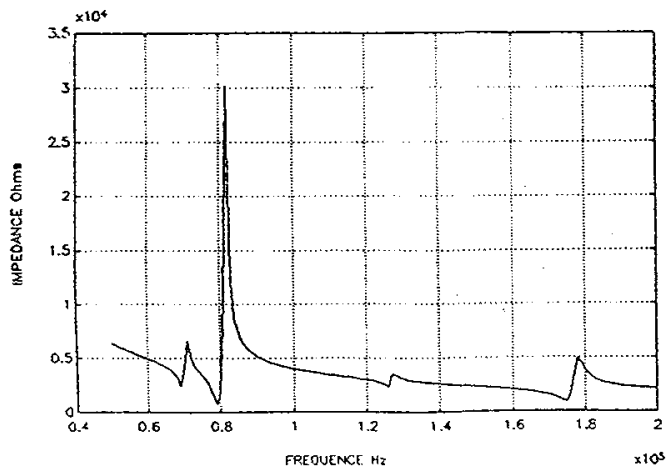

Fig. 4 Courbe simulée de l'impédance du seçeur du moteur correspondant à la figure 3 